\title{
SDSS IV MaNGA: Star-formation-driven Biconical Outflows in the Local Universe
}

\author{
Dmitry Bizyaev ${ }^{1,2,11}$ (1), Yan-Mei Chen ${ }^{3,4}$, Yong Shi ${ }^{3,4}$ (1), Rogemar A. Riffel ${ }^{5,6,7}$ (1) , Rogerio Riffel ${ }^{5,8}$, \\ Aleksandar M. Diamond-Stanic ${ }^{9}$, and Namrata Roy ${ }^{10}$ (i) \\ ${ }^{1}$ Apache Point Observatory and New Mexico State University, Sunspot, NM, 88349, USA \\ ${ }^{2}$ Sternberg Astronomical Institute, Moscow State University, Moscow, Russia \\ ${ }^{3}$ School of Astronomy and Space Science, Nanjing University, Nanjing 210093, People's Republic of China \\ ${ }^{4}$ Key Laboratory of Modern Astronomy and Astrophysics (Nanjing University), Ministry of Education, Nanjing 210093, People's Republic of China \\ ${ }^{5}$ Laboratório Interinstitucional de e-Astronomia, 77 Rua General José Cristino, 20921-400, Rio de Janeiro, Brazil \\ ${ }^{6}$ Universidade Federal de Santa Maria, Departamento de Física, Centro de Ciências Naturais e Exatas, 97105-900, Santa Maria, RS, Brazil \\ 7 Department of Physics \& Astronomy, Johns Hopkins University, Bloomberg Center, 3400 N. Charles St, Baltimore, MD 21218, USA \\ ${ }^{8}$ Instituto de Física, Universidade Federal do Rio Grande do Sul, Campus do Vale, 91501-970 Porto Alegre, Brazil \\ ${ }^{9}$ Bates College, Department of Physics \& Astronomy, 44 Campus Avenue, Carnegie Science Hall, Lewiston, ME 04240, USA \\ ${ }^{10}$ Department of Astronomy and Astrophysics, University of California, 1156 High Street, Santa Cruz, CA 95064, USA \\ ${ }^{11}$ Special Astrophysical Observatory of the Russian AS, 369167, Nizhnij Arkhyz, Russia \\ Received 2019 March 29; revised 2019 July 13; accepted 2019 July 18; published 2019 September 11
}

\begin{abstract}
We present a sample of 48 nearby galaxies with central, biconical outflows identified by the Mapping Nearby Galaxies at APO survey. All considered galaxies have star-formation-driven biconical (SFB) central outflows, with no signs of an active galactic nucleus. We find that the SFB outflows require high central concentration of the star formation rate. This increases the gas velocity dispersion over the equilibrium limit and helps maintain the gas outflows. The central starbursts increase the metallicity, extinction, and the $[\alpha / \mathrm{Fe}]$ ratio in the gas. A significant amount of young stellar population at the centers suggests that the SFBs are associated with the formation of young bulges in galaxies. More than $70 \%$ of SFB galaxies are group members or have companions with no prominent interaction, or show asymmetry of external isophotes. In 15\% of SFB cases, stars and gas rotate in the opposite directions, which points at the gas infall from satellites as the primary reason for triggering the SFB phenomena.
\end{abstract}

Key words: galaxies: spiral - galaxies: star formation - ISM: jets and outflows - ISM: kinematics and dynamics

\section{Introduction}

Biconical outflow of gas from the centers of galaxies is a typical manifestation of processes powered by active galactic nuclei (AGNs). Gas outflows can also be powered by intensive star formation and driven by supernovae and stellar winds from young stellar populations (Strickland \& Heckman 2007). They are found to be ubiquitous in galaxies at low (Veilleux et al. 2005; Chen et al. 2010) and high redshifts (Davies et al. 2018; Rubin et al. 2014). Intensive outflows driven by star formation bursts in galaxies without AGNs found in nearby starburst galaxies were dubbed superwinds (Lehnert \& Heckman 1995, 1996b; Heckman et al. 2015).

The first time the superwinds were detected in M82 (Lynds \& Sandage 1963; Burbidge et al. 1964), it was as a gas outflow from the center. In most cases of superwinds, the warm gas escapes the galaxies in all directions (Lehnert \& Heckman 1996b), whereas just a few local galaxies, e.g., M82 and NGC 253, demonstrate biconical star-formation-driven superwinds stemmed from the centers (Heckman et al. 1990; Lehnert et al. 1999). A large mass of warm gas is ejected in superwinds (Heckman 2002; Chisholm et al. 2015) at a high rate $\left(10-20 M_{\odot} \mathrm{yr}^{-1}\right)$ to the distance of a dozen kpc (Veilleux et al. 2003; Strickland \& Heckman 2007) with a hundred (Shopbell \& Bland-Hawthorn 1998; Veilleux et al. 2005) to thousand $\mathrm{km} \mathrm{s}^{-1}$ speed (Heckman et al. 2000) when the star formation surface density exceeds a threshold for the superwinds of $0.1 M_{\odot} \mathrm{yr}^{-1} \mathrm{kpc}^{-2}$ (Heckman 2002). Numerical simulations predict the ubiquity of the central biconical outflows (Tenorio-Tagle \& Muñoz-Tuñón 1998; Fielding et al. 2017; Schneider et al. 2018).

Galactic winds help inhibit star formation and remove baryonic matter from star formation sites, which affects the chemical evolution in galaxies, shape integral scaling relations (Veilleux et al. 2005; Fielding et al. 2017) and enrich the intergalactic gas media. In turn, the outflows may remove the gas from the galaxies only temporarily (Oppenheimer \& Dave 2006; Oppenheimer et al. 2010; Leroy et al. 2015), which makes them an important component of the extragalactic gas supply circulation process. The centrally concentrated starformation-driven outflow provides a simplified case with clear central localization. Exploring the reasons for starting and maintaining the central, biconical outflow helps to better understand processes of gas exchange between the highaltitude intergalactic medium and low-altitude sites of active star formation.

Statistical power of large integral field spectroscopic surveys allows us to look for more examples of the centrally concentrated outflows in the local Universe (Roche et al. 2015; Ho et al. 2016; Gallagher et al. 2019) and to determine the key factors that play a role in the life cycle of the outflows (Veilleux et al. 2005; Zhang 2018). In this paper we focus on observational manifestation and properties of the central, biconical star-formation-driven outflows identified in a large sample of galaxies. We explain our selection procedure and the outflow galaxies sample formation criteria in Section 2. We also create a sample of regular galaxies, without noticeable outflows, which we use for comparison purposes. The observational properties of the galaxies in the samples are examined in Section 3. In Section 4 we explore the differences between the galaxies with detected outflows and regular galaxies, and discuss unique features of the galaxies with the central, star-formation-driven outflows. 


\section{The Sample of Star-formation-driven Bicones from MaNGA Observations}

We look for the star formation bicones (SFB) in a large sample of relatively nearby galaxies using results from the Mapping Nearby Galaxies at APO (MaNGA; Bundy et al. 2015) survey performed at a dedicated $2.5 \mathrm{~m}$ telescope (Gunn et al. 2006). MaNGA, a part of the Sloan Digital Sky Survey (SDSS; York et al. 2000; Blanton et al. 2017), is a multiple (Drory et al. 2015; Law et al. 2016) Integral Field Unit survey of several thousand local galaxies (median redshift $\sim 0.03$; Yan et al. 2016) with a spectral resolution of 2000 and a $3600-10300 \AA$ wavelength coverage (Smee et al. 2013). The survey's target selection (Wake et al. 2017) provides a roughly uniform stellar mass distribution for MaNGA galaxies and allows us to obtain kiloparsec-scale spatial resolution maps of the stellar and ionized gas kinematics (Law et al. 2015).

We started with the MaNGA "product launch" MPL-6 (Law et al. 2016) which released 4857 objects, estimated the inclination from the ellipticity of SDSS images as in Chen et al. (2010), and selected a sample of 1589 galaxies with the inclination of $60^{\circ}$ or higher. Next, we selected only the galaxies in which the central and eight adjacent spaxels fall onto the star formation region on the Baldwin-Phillips-Terlevich (BPT) diagram (Baldwin et al. 1981). Only the spaxels with the signal-to-noise ratio $(\mathrm{S} / \mathrm{N})$ of 3 and greater in all significant for this study emission lines $(\mathrm{H} \alpha, \mathrm{H} \beta$, [N II] $\lambda 6584$, and [O III] $\lambda 5007)$ were considered. As an independent check, we check the galaxy BPT classification made in the MPA-JHU catalog (Tremonti et al. 2004), which uses SDSS spectroscopy data, independent of MaNGA. All SFB sample and control galaxies were confirmed to have star-forming centers in the MPA-JHU catalog.

As one more verification, we place all considered galaxies on the star formation rate (SFR)-stellar-mass diagram, see Figure 1 and ensure that the objects with and without the SFB occupy the same region, which corresponds to the star formation, blue cloud. We inspect two-dimensional maps of the $\mathrm{H} \alpha$ and [O III] $\lambda 5007$ equivalent width (EW) and maps of the gas velocity dispersion in all galaxies. Note that we identify the extra-planar ionized gas structures similar to those reported by Cheung et al. (2016), Roy et al. (2018), and Riffel et al. (2019), who notice ionized gas regions with enhanced $\mathrm{H} \alpha$ and $[\mathrm{O}$ III] $\lambda 5007$ emission extended along the minor axes in a sample of early-type galaxies. We split the galaxies by three groups: first one with the biconical structures aligned along the minor axis in the emission lines; second group without these features, and the third group with uncertain or disturbed maps of the $\mathrm{H} \alpha$ and $[\mathrm{O} \mathrm{III}] \lambda 5007 \mathrm{EW}$. The latter group also includes galaxies with perturbed emission line fields, for which we cannot claim a clearly identified SFB. The gas velocity dispersion fields were inspected for the galaxies with prominent biconical structures. All these galaxies demonstrate enhanced velocity dispersion with respect to the galactic periphery.

Our inspection of the gas and stellar kinematic maps revealed seven SFB galaxies with counter-rotating gas and stars, which means close to a $180^{\circ}$ difference in the spin vectors between them in the case of edge-on galaxies. We also identified several cases of perpendicular rotation between gas and stars, and rejected such objects from the samples as polar ring galaxies. Any interacting or warped objects, as well as members of tight groups or pairs, were removed from all
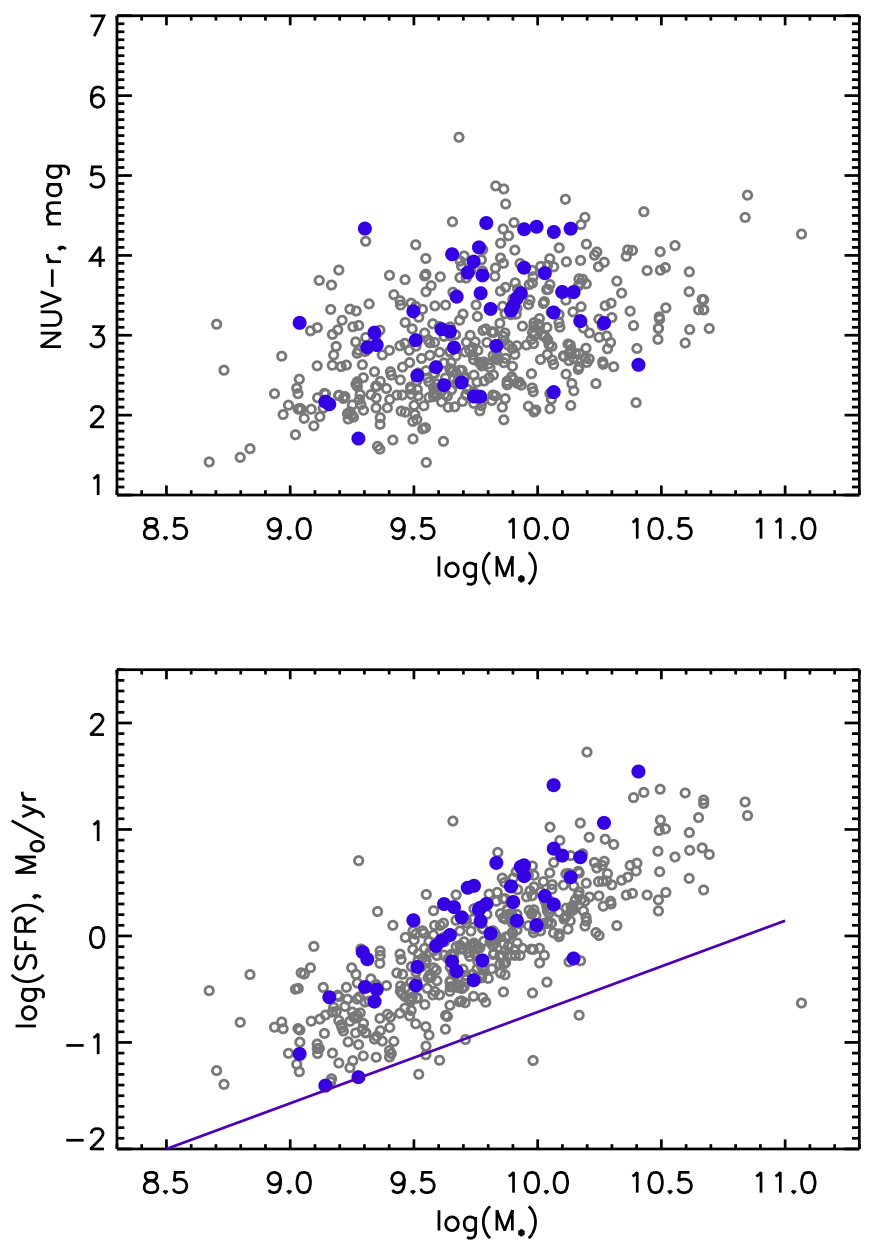

Figure 1. MaNGA galaxies with (blue bullets, SFB sample, see text) and without (gray circles, control sample, see the text) the star-forming biconical structures on the SFR-stellar-mass diagrams. The stellar mass is taken from the NASA-Sloan Atlas (Blanton et al. 2011). Top: the near-ultraviolet-red magnitude difference estimated by the NASA-Sloan Atlas. Bottom: the integrated star formation rate in the galaxies from the MaNGA data, see Section 3.1. The solid line designates the demarcation between the star-forming and green valley galaxies according to Chang et al. (2015).

samples. We tailor the control sample of galaxies such that the galaxies with and without SFB have very similar distribution by their distances, stellar mass, and Sérsic index. The first group forms the target sample of 48 galaxies with biconical outflow (SFB). From the second group we selected the galaxies in the radial velocity range and stellar mass (according to the NASA-Sloan Atlas (NSA) catalog) similar to the target sample (5000 and $14,000 \mathrm{~km} \mathrm{~s}^{-1}$ ), and thus formed the control sample of 432 objects, which we use for comparison purposes. Thus, the control galaxies are similar to the SFB objects, but do not show enhanced emission along the minor axis. We removed the third group of 61 uncertain galaxies from further consideration. An example of our galaxies with star formation bicones is shown in Figure 2. The MaNGA galaxy 8448-3701 shown in Figure 2 has starforming only spaxels in the central area. The optical SDSS image looks rather regular, but the emission line maps demonstrate clear structures aligned along the minor axis of the galaxy, in which the gas velocity dispersion is also higher than in the rest of the galaxy. 

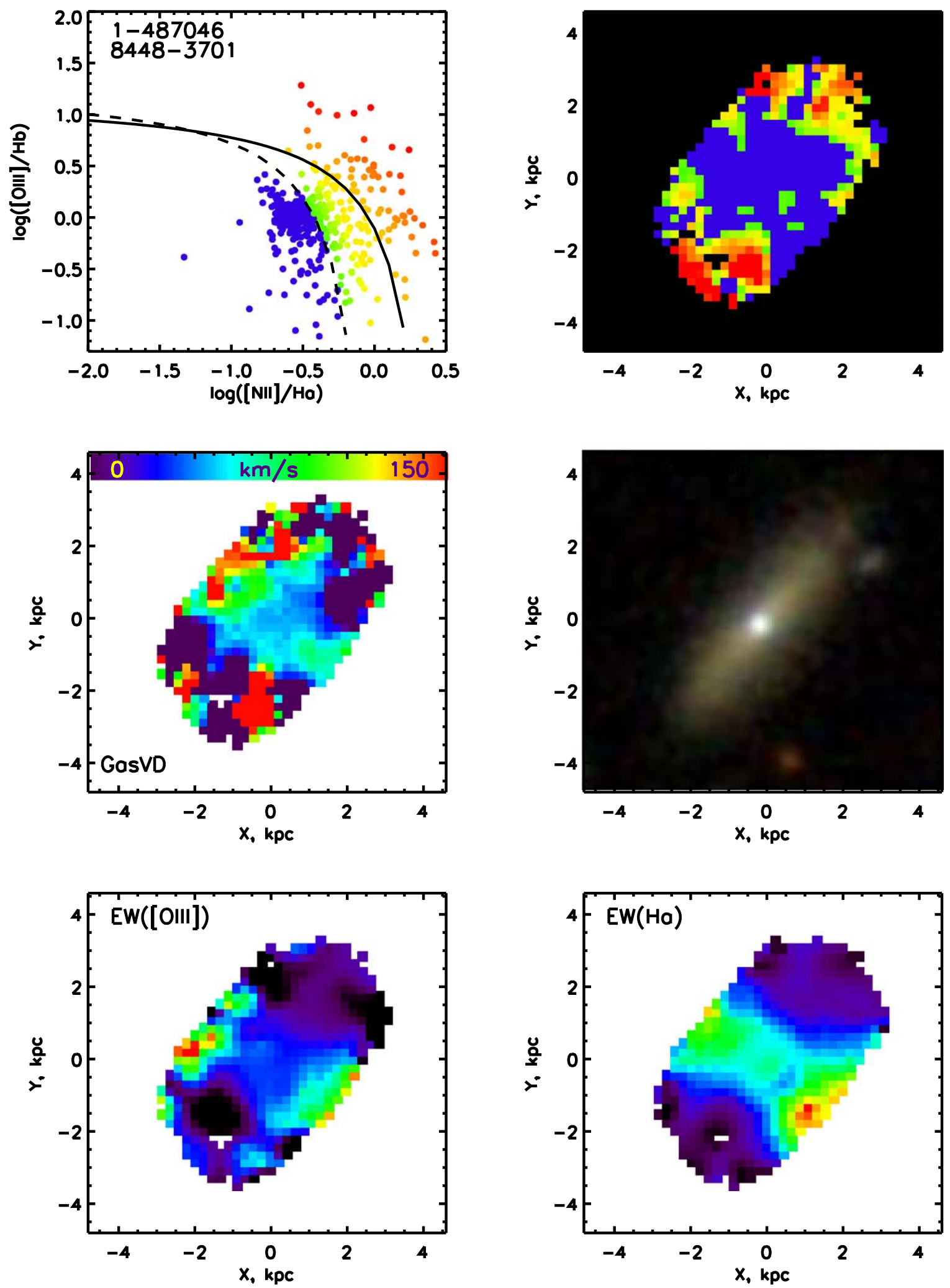

Figure 2. One of our galaxies, 8448-3701. Top left: a BPT diagram for MaNGA spaxels with $\mathrm{S} / \mathrm{N}>5$ in the H $\alpha$ flux. The colors designate the star formation (blue), AGN/LINER (red) and composite (yellow/green) spaxels. The demarkation curves by Kewley et al. (2001) and Kauffmann et al. (2003) separate the star formation, AGN, and composite regions. Top right panel: the distribution of the color coded BPT spaxels over the galaxy. Middle left panel: the ionized gas velocity dispersion map. Middle right panel: SDSS color image of the galaxy. Bottom left panel: the [O III] equivalent width (EW) map of the galaxy. The emission distribution reveals the biconical outflow along the minor axis of the galaxy associated with high gas velocity dispersion. Bottom right panel: the H $\alpha$ EW map of the galaxy. 


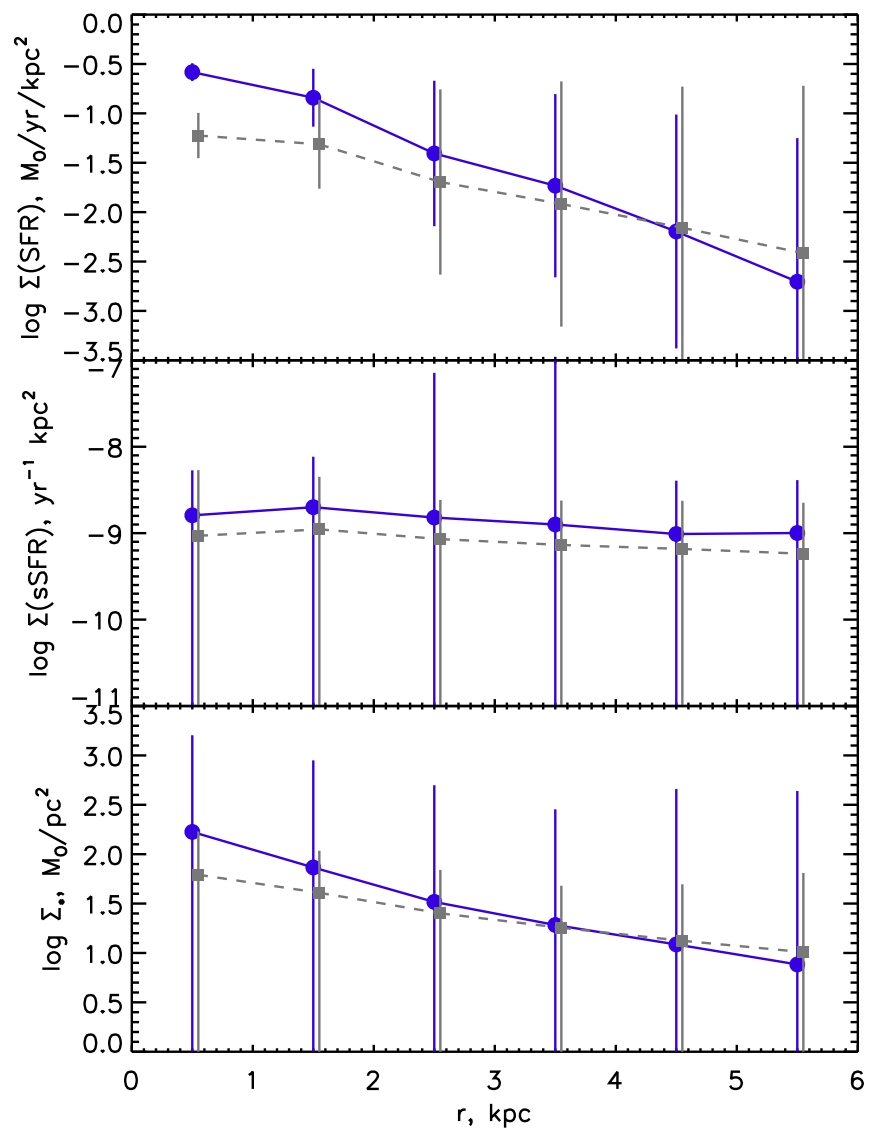

Figure 3. Median values for the star formation rate surface density (top), specific star formation rate surface density (middle), and stellar surface density (bottom) for the SFB galaxies (blue symbols and solid lines) and control sample (gray symbols and dashed lines). The error bars reflect the $1 \sigma$ standard deviation of the objects in each bin.

\section{Observational Properties of the Galaxies with Star- formation-driven Bicones}

\subsection{The Star-formation Rate and the Extinction}

The galactic effective radii, Sérsic index, and estimated stellar masses are adopted from the $\mathrm{NSA}^{12}$ (Blanton et al. 2011). Although the SFB galaxies have similar stellar mass and distance distribution, some of their features differ significantly from those in the control sample. We compare the median radial profiles of the star formation rate, specific star formation rate, and stellar surface density for the SFB and control sample in Figure 3 and find that only the star formation surface density is significantly different in the SFB galaxies, and only in their central regions.

The internal extinction $A_{V}$ in the galaxies is estimated through the Balmer decrement via $\mathrm{H} \alpha / \mathrm{H} \beta$ ratio with the assumption of Case B recombination (Osterbrock 1989) extinction-free ratio of 2.86 and Cardelli et al. extinction law (Cardelli et al. 1989).

Each galaxy is split by elliptical annuli $1 \mathrm{kpc}$ wide if the inclination was less than $80^{\circ}$, or by zones of the same width if the inclination was higher. The reported $A_{V}$ is an average value in each zone or annulus. Only the spaxels with no bad reduction flags and $\mathrm{S} / \mathrm{N}>3$ in the $\mathrm{H} \alpha$ and $\mathrm{H} \beta$ flux are used for the $A_{V}$ calculations. The same elliptical annuli or zones

\footnotetext{
${ }^{12}$ http://nsatlas.org
}

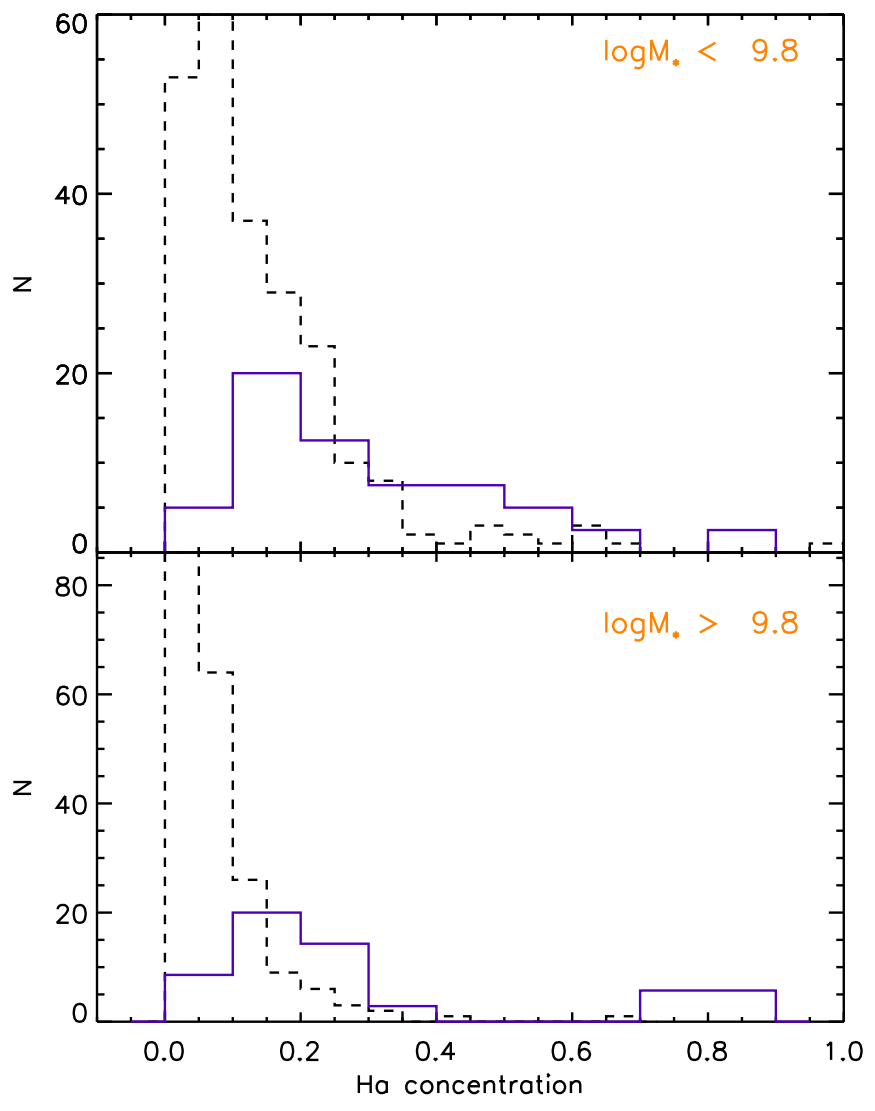

Figure 4. Central concentration of $\mathrm{H} \alpha$ surface brightness for the SFB (blue solid) and control (black dashed) galaxies in the low (top) and high (bottom) mass galaxies.

were used to estimate the star formation rate from the extinction corrected $\mathrm{H} \alpha$ surface brightness (Martin \& Kennicutt 2001). The $\mathrm{H} \alpha$ surface density concentration is estimated as the ratio of $\mathrm{H} \alpha$ luminosity within and outside the central $1 \mathrm{kpc}$ circle.

We split all galaxies into two groups with low and high stellar masses (corresponding to $\log \left(M_{*} / M_{\odot}\right)$ under or over 9.8 dex). The central surface density of star formation is higher in the SFB galaxies of all stellar mass groups. The $\mathrm{H} \alpha$ luminosity concentration is also prominently higher in the SFB galaxies with respect to the regular ones, see Figure 4. These trends suggest that the star formation is the primary mechanism that powers up the gas outflow, in agreement with Heckman (2002).

The galactic extinction $A_{V}$ in the SFB galaxies is higher than that in the control sample, on average, especially in the galaxies in the lower mass bin $\left(\log \left(M_{*} / M_{\odot}\right)<9.8 \mathrm{dex}\right)$. The median central $A_{V}$ is 2.31 and $1.59 \mathrm{mag}$ in the SFB and control samples, respectively, with the sigma (estimated as 1.48 of the median absolute deviation) of 0.96 and 1.05 mag for the same samples.

\subsection{The Gas Kinematics}

The [S II] $\lambda 6717,6731$ line ratio allows us to estimate the ionized gas density (Osterbrock 1989; Perez-Montero 2017) at the centers of SFB galaxies. We assume that the gas density decreases exponentially with the distance to the galactic midplane, see e.g., Bizyaev et al. (2017) and Levy et al. (2019).

The ionized gas velocity dispersion at the central outflow area is enhanced in the SFB galaxies. Its typical amplitude in our sample is of the order of $100 \mathrm{~km} \mathrm{~s}^{-1}$, and the central and 
minor axis velocity dispersion noticeably exceeds that in the rest of the galaxy. The gas emission line profiles estimated along the minor axes of the galaxies are systematically wider in the SFB galaxies. The maximum minor axes gas velocities are $276 \mathrm{~km} \mathrm{~s}^{-1}$ in the SFBs, on average, versus the $165 \mathrm{~km} \mathrm{~s}^{-1}$ in the regular galaxies from the control sample. These values are estimated for the gas on the minor axis (within $0.5 \mathrm{kpc}$ from it) at the altitudes above $1 \mathrm{kpc}$ from the galactic midplane. At the same time, this is problematic to estimate the gas outflow velocities accurately for our highly inclined galaxies; the identified outflows are often shaped as very-narrow bisymmetric structures, with small opening angle. Small uncertainties of the opening angle lead to large errors in the outflow velocity. We assume that the gas escapes from galaxies if its velocity dispersion is increased due to the energy injection from supernovae and young stellar winds. The latter can be determined from the star formation surface density $\Sigma_{\mathrm{SFR}}$ as $v \sim \Sigma_{\mathrm{SFR}}^{\gamma}$, where the $\gamma$ can range from 0.18 to 1 depending on the primary reason for the gas turbulence (e.g., stellar and supernovae feedback, Krumholz \& Burkhart (2016) and Dib et al. (2006), or gravitational instability, Krumholz \& Burkhart (2016) and Lehnert et al. (2009)).

We estimate the bicone size in the galactic midplane from the MaNGA [O III] $\lambda 5007$ and $\mathrm{H} \alpha$ images. The typical in-plane bicone diameter is $2 \mathrm{kpc}$. We assume that the gas density is an order of magnitude less at the escape altitude than at the midplane. The mass of the gas at the center $M_{g c}$ is coarsely estimated under the assumption that all gas is ionized and fills a cylindric volume (Heckman et al. 1990), whose diameter equals to the size of the bicone in the galactic midplane, and the height is $1 \mathrm{kpc}$ in all galaxies.

The equilibrium central velocity dispersion in the galaxies is estimated by van der Kruit \& Freeman (2011) as

$$
\sigma_{c}=e^{1 / 2}\left(0.33 v_{c}-2\right),
$$

where the $v_{c}$ is the maximum circular velocity in the galaxy. We assume that the gas starts forming the central outflow when its velocity dispersion exceeds the $\sigma_{c}$.

Large uncertainties in the direct measurement of the gas velocity dispersion in highly inclined galaxies make us consider the central surface density of the star formation $\Sigma_{\mathrm{SFR}}$, which is expected to be connected to the gas velocity dispersion driven by the star formation feedback as $\sigma_{g} \sim \Sigma_{\text {SFR }}^{1 / 2}$ (Krumholz \& Burkhart 2016). Figure 5 compares the the $\Sigma_{\mathrm{SFR}}$ (in $M_{\odot} \mathrm{yr}^{-1} \mathrm{kpc}^{-2}$ ) with the gas velocity dispersion expected in the disks of galaxies in equilibrium, Equation (1). The blue line in Figure 5 corresponds to the case of $\sigma_{g}=200 \Sigma_{\mathrm{SFR}}^{1 / 2}\left[\mathrm{~km} \mathrm{~s}^{-1}\right]$. We draw the line such that it places all massive SFB galaxies (bottom) and all but one low massive SFB galaxies (top) to the right side of the line. The comparison suggests that the star formation rate in our SFB galaxies makes the gas central velocity dispersion much higher than required for the equilibrium in the galaxies, in contrast with the regular galaxies.

\subsection{The Gas Metallicity}

Similar to the extinction, the strong emission line ratios in elliptical annuli corrected for the internal extinction via $A_{V}$ were used to estimate the gas metallicity. In all cases we required that fluxes in all emission lines had $\mathrm{S} / \mathrm{N}>3$ and there are no bad reduction flags at the utilized spaxels. We consider three different metallicity calibrations for the gas metallicity $12+\log (\mathrm{O} / \mathrm{H})$ : N2S2Ha (Dopita et al. 2016), O2N2 (Kewley \& Dopita 2002),

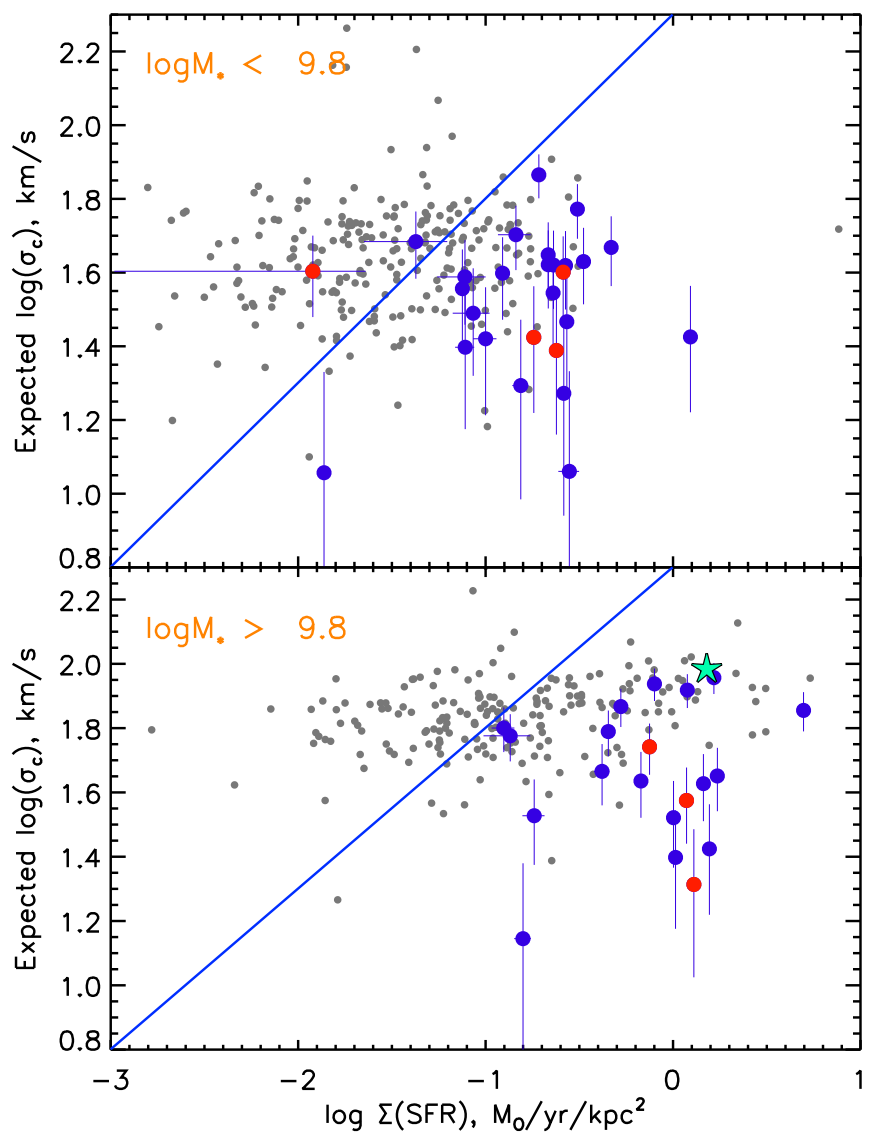

Figure 5. Central velocity dispersion in the case of equilibrium $\left(\sigma_{c}\right.$, from Equation (1)) in the SFB (blue bullets) and control (gray dots) galaxies in dependence of the SFR surface density at the center. The red bullets mark the SFB galaxies with counterrotation between the gas and stars. The green star designates the galaxy M82. The solid line corresponds to the case of the equality of $\sigma_{c}$ to the star-formation-driven gas velocity dispersion $\sigma_{g}=200 \Sigma_{\mathrm{SFR}}^{1 / 2}$ at the center (see the text). The error bars indicate the $2 \sigma$ uncertainty for the SFB objects.

and PG16 (Pilyugin \& Grebel 2016). The first two reveal the same radial distributions in our galaxies, although with slightly different zero-point. We use the N2S2Ha calibrator throughout of the paper because it uses red emission lines at wavelengths near to each other and thus is almost independent of the internal extinction. We notice that the PG16 calibrator demonstrates similar radial distributions but shows larger uncertainties in the abundances, most probably due to the use of blue and red lines in a mix, which allows uncertainties in the $A_{V}$ to affect the estimated metallicity strongly. Note that our conclusions based on the same metallicity calibration technique for the SFB and control sample should be mostly independent of the chosen metallicity calibrator's internal accuracy.

Comparison of the radial distributions of the gas metallicity reveals different trends for the low- and high-mass galaxies. The low mass SFB galaxies have 0.25 dex higher central metallicities $12+\log (\mathrm{O} / \mathrm{H})$ than the control galaxies of the same mass, see Figure 6. The comparison for the N2S2Ha calibrator is shown, but the other considered metallicity calibrators show similar differences. In this case we observe the outflow of gas enriched by the supernovae explosions. The difference in the metallicities and extinction $A_{V}$ also leads to a more efficient gas removal from the galactic midplane with the 


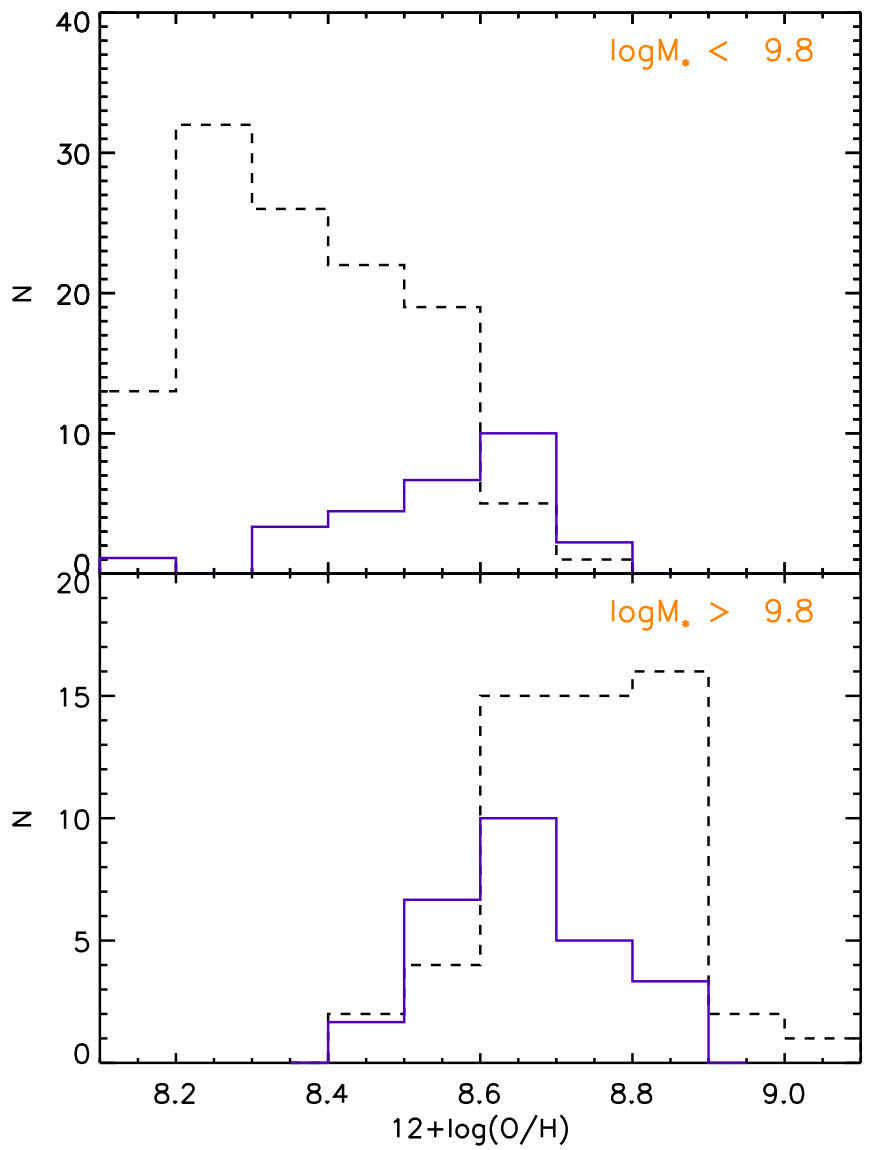

Figure 6. Gas metallicity $12+\log (\mathrm{O} / \mathrm{H})$ (from the $\mathrm{N} 2 \mathrm{~S} 2 \mathrm{Ha}$ calibrator) at the central regions of the SFB (blue solid line) and control (black dashed line) galaxies of low (top panel) and high (bottom panel) stellar mass.

energy and radiation injected by the star formation process. In contrast, massive SFB and control galaxies have similar, slightly subsolar gas metallicity.

We also notice that the central gas in the low and high-mass SFB galaxies has similar $12+\log (\mathrm{O} / \mathrm{H})$ metallicity that varies narrowly with respect to the control sample ranges, around 8.6 dex. Because of the similar star formation density threshold for starting the central outflow in all SFB galaxies, one may expect the narrow metallicity distribution similarity of the initial, preenriched gas in them. In turn, it points toward the external origin of the gas, which can infall from larger galaxies in groups, like in the case of M82, where a larger galaxy M81 supplies the gas to the M82 biconical outflow (Lehnert et al. 1999).

\subsection{Stellar Population}

The main MaNGA data reduction pipeline (Law et al. 2016) reports all Lick indices (Worthey \& Ottaviani 1997), their uncertainties, and data quality flags. We study the radial distributions of age and metallicity indicators determined from the absorption spectra features averaged over the $1 \mathrm{kpc}$ wide elliptical annuli or zones in the same way as described above. We took into account only those spaxels where the indices have $\mathrm{S} / \mathrm{N}>3$ and the data reduction flags are good. We use the D4000 index (Gorgas et al. 1999) as the average stellar population age indicator. For the iron abundance we use the $\langle\mathrm{Fe}\rangle=$ $\mathrm{Fe} 5270+\mathrm{Fe} 5335$ combination. The $[\alpha / \mathrm{Fe}]$ is estimated using

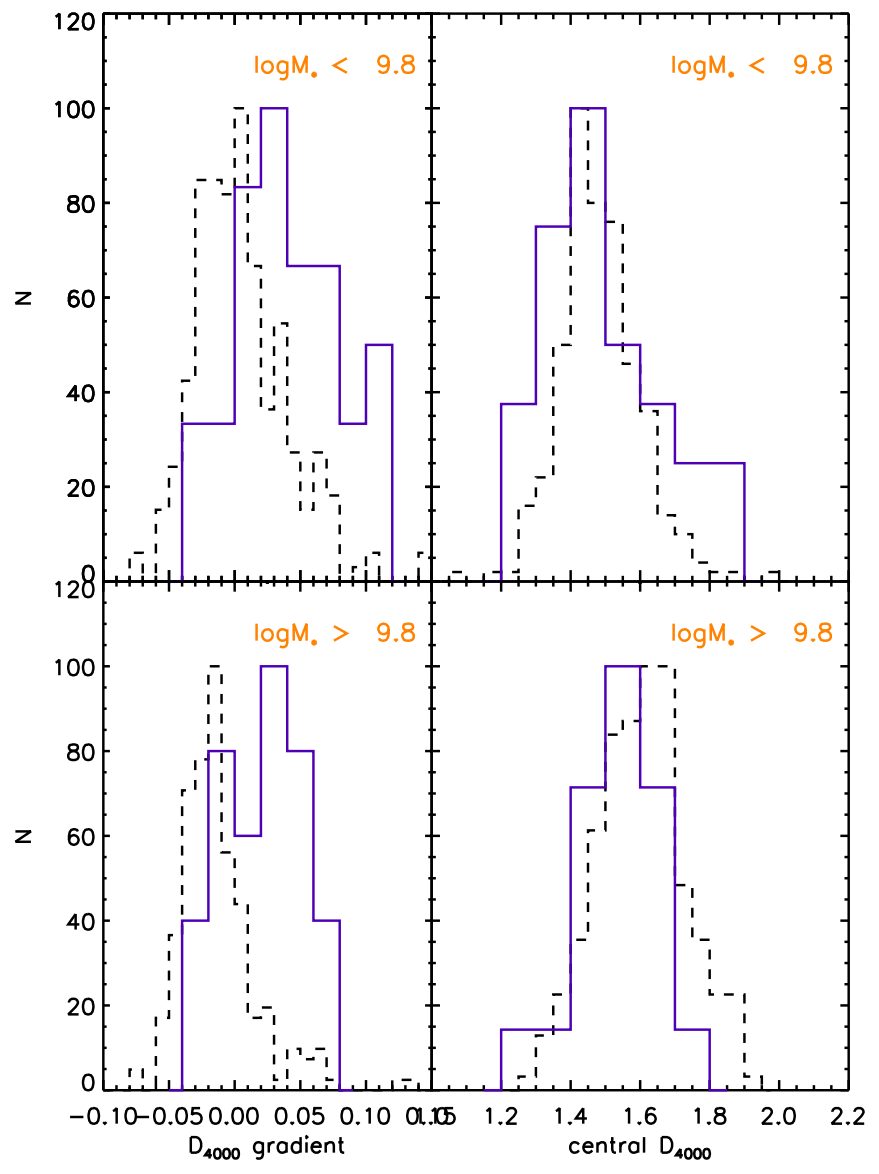

Figure 7. Central values and gradients of the D4000 index for the SFB (blue solid) and control (black dashed) galaxies of low (top panel) and high (bottom panel) stellar mass. The peaks of all distributions are normalized by 100 .

the strongest $\mathrm{Mg}$ feature $\mathrm{Mgb}$ as $[\alpha / \mathrm{Fe}]=-1.030+1.016 X-$ $0.141 X^{2}$, where $X=\mathrm{Mg} \mathrm{b} /\langle\mathrm{Fe}\rangle$, which is a fit to the relation by Thomas et al. (2002).

Figure 7 contrasts the central value and gradient of the D4000 index in the SFB and control galaxies. The D4000 sensitive to the age of stellar population is not different at the centers, while the D4000 radial gradient is high positive in SFB hosts, in a contrast with mostly zero or negative gradients in the regular galaxies. Since D4000 traces the efficient age of underlying stellar population, it suggests a sharp concentration of significant population of young stars in the SFBs. Such a peak of young objects at the centers points toward a prior infall of building material (gas) to the central region.

Comparison of effective metallicity of the stellar population from the Fe5270 and Fe5335 indices do not show a significant difference for the SFB galaxies. At the same time, the $[\alpha / \mathrm{Fe}]$ estimated from a combination of $\mathrm{Mgb}$ and $\mathrm{Fe}$ indices reveals high fraction of alpha-enhanced centers of SFB galaxies, see Figure 8 . The latter fact indicates a relatively short age (much less than $1 \mathrm{Gyr}$ ) of the central starburst due to a time lag of the supernovae (McWilliam 1997).

\section{Discussion}

Contrasting the large sample of the SBF and regular galaxies allows us to notice differences in observational properties of these galaxies and to make conclusions about the distinctive features of the SFB galaxies. 


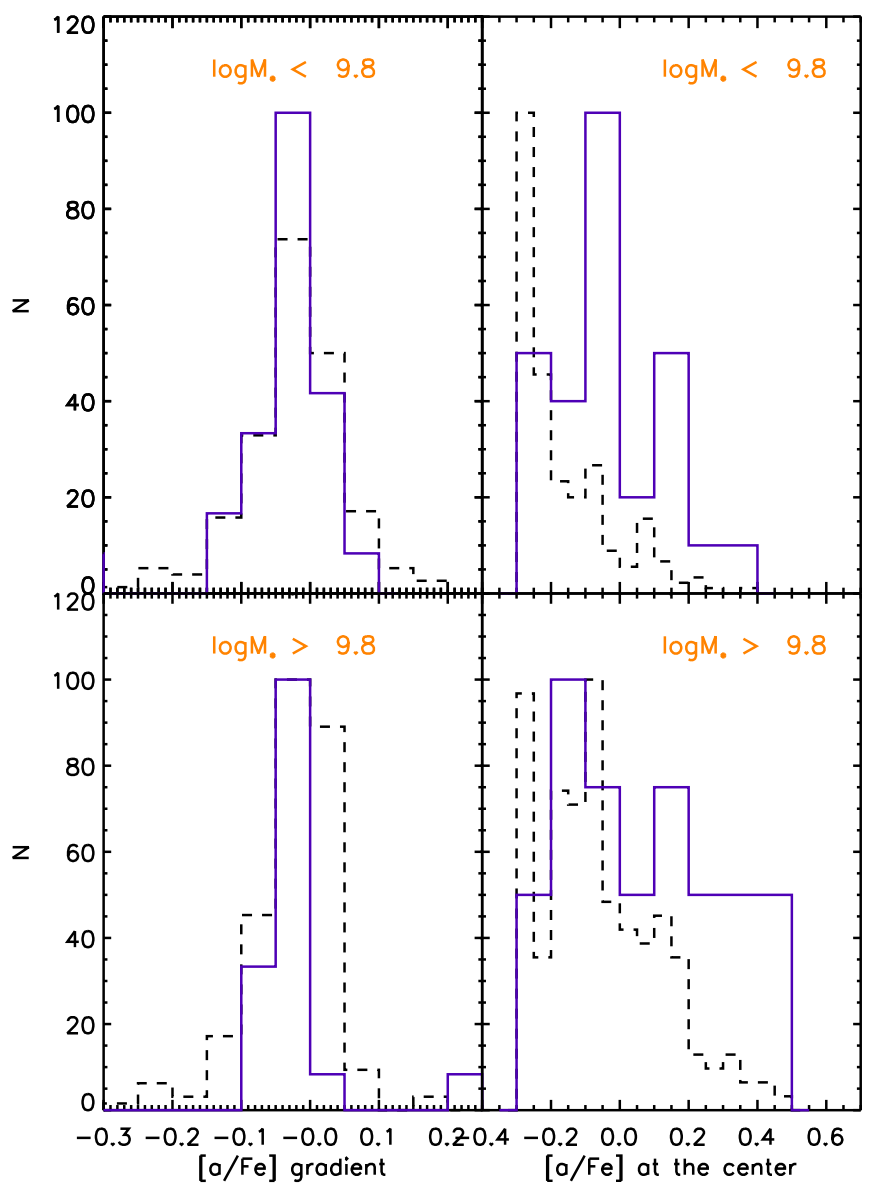

Figure 8. Central values and gradients of the $[\alpha / \mathrm{Fe}]$ for the SFB (blue solid) and control (black dashed) galaxies of the low and high-mass ranges (top and bottom panels, respectively). The peaks of all distributions are normalized by 100 .

The driver of the central outflows. The enhanced SF surface density at the center is a distinctive feature of the galaxies with biconical outflows (Figure 3). We compare the SF-driven velocity dispersion in the ionized gas with the equilibrium velocity dispersion in Figure 5 and conclude that the gas must expand inn the direction perpendicular to the galactic midplane and finally escape to high galactic altitudes. A good illustration of the rapid and centrally localized starburst in the SFBs comes from a systematically high radial gradient of D4000 index, see Figure 7, which suggests a large difference of stellar age along the radius in the SFB galaxies; they have a much younger stellar population at the center than at the outer regions. At the same time, the centers of SFBs demonstrate enhanced abundance of alpha-elements traced by the $[\mathrm{Mg} / \mathrm{Fe}]$ indicator. These facts suggest that the rapid star formation started much less than $1 \mathrm{Gyr}$ ago, and that since that time it has built a significant amount of new stars sufficient to make the photometric profiles steeper and the overall effective radii shorter.

Important conditions for creating and maintaining the SFBs. We notice systematically higher $A_{V}$ and gas metallicity in the low mass SFB galaxies with respect to the regular ones (Figure 6). The higher gas extinction helps couple the radiation with gas, and to push the gas from the galaxy more efficiently (see Zhang 2018 and references therein). These factors also may be responsible for the star formation density threshold decreasing (Heckman 2002), responsible for the outflow emergence below the $0.1 M_{\odot} \mathrm{yr}^{-1}$ in small galaxies, see Figure 5. We consider
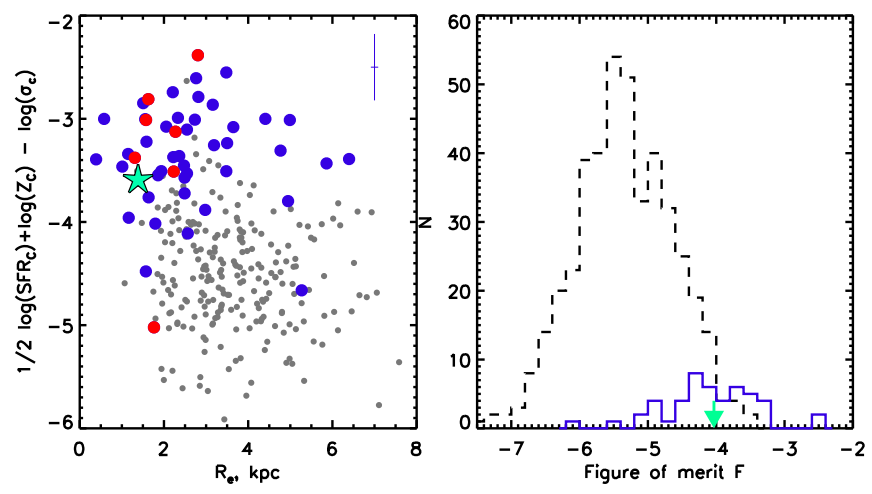

Figure 9. Left: the ratio of the star formation driven to the equilibrium velocity dispersions versus the effective radius $R_{e}$. The former gas velocity dispersion is estimated in arbitrary units as $\Sigma_{\mathrm{SFR}}^{1 / 2} Z_{c}$, where $Z_{c}=40 \cdot 10^{(\mathrm{O} / \mathrm{H})} \tau_{V}$ (see the text). The blue bullets denote the SFB galaxies, the red bullets highlight the SFB galaxies with a counterrotation between the gas and stars. The gray dots show the control sample. The error bars at the top right corner indicate the typical uncertainty. Right: the figure of merit $F=\log \left[\Sigma_{\mathrm{SFR}}^{1 / 2} Z_{c}\right]-\log \left(\sigma_{c}\right)-2 \log R_{e}$ in the SFB (blue solid line) and control (gray dashed line) galaxies. The green arrow designates the galaxy M82.

the ratio of the estimated and the equilibrium gas velocity dispersions with an addition of empirical dependence on the metallicity and extinction, $f=\log \left[\Sigma_{\mathrm{SFR}}^{1 / 2} Z / \sigma_{c}\right]$, where the SFR surface density $\Sigma_{\mathrm{SFR}}$ is measured at the center from the $\mathrm{H} \alpha$ luminosity, $Z=\tau_{V} 40 * 10^{(\mathrm{O} / \mathrm{H})}, \tau_{V}$ is the optical depth from the extinction $A_{V}, \sigma_{c}$ is the equilibrium velocity dispersion at the center of galaxy from Equation (1), and the gas velocity dispersion is assumed to be driven by the star formation feedback (Krumholz \& Burkhart 2016), for which $\sigma \sim \Sigma_{\text {SFR }}^{1 / 2}$. Figure 9 (left) shows the comparison of the ratio $f$ with the effective radii $R_{e}$ for the galaxies in our samples. By introducing the empirical value $f$ we attempt to find a combination of parameters suitable for selecting the SFB galaxies. The SFB galaxies are rather well separated from the regular ones in Figure 8 (left), which suggests the importance of of the factors contributing to the $f$ for maintaining the SFBs. Figure 9 (right) shows the distribution of the figure of merit $F$ introduced as $F=f-2 \log R_{e}$. Using $F$, we can efficiently separate the SFB galaxies from the regular ones.

The central concentration of the outflows. If the gas outflow condition $\left(\sigma_{g}>\sigma_{c}\right.$, Figure 5) was fulfilled in the whole galaxy, the gas would escape from all regions of the galactic body. The central localization of the outflow is caused by the concentration of star formation to the center. The SFB galaxies have systematically shorter $R_{e}$ than the control galaxies. In turn, the ignition of the powerful central starburst in the SFB galaxies is impossible without driving a large amount of gas at the center, sufficient for creating a significant amount of your stars, which creates central concentration of young stellar population.

The lifetime of the $S F B$ s. We estimate the outflow rate $\dot{M}_{\text {out }}$ in our SFB galaxies using the central electron density as $\dot{M}_{\text {out }}=$ $1.4 \pi m_{p} v_{\text {out }} n_{e} r_{c}^{2}$, where $m_{p}$ is the mass of proton, $n_{e}$ is the electron density, $r_{c}$ is the bicone radius (see Section 3.2), $v_{\text {out }}$ is estimated as $v_{\text {out }}=140 \Sigma_{\text {SFR }}^{1 / 2}$ (Krumholz \& Burkhart 2016; Yu et al. 2019), and the factor 1.4 accounts for the contribution of heavy elements to the total gas mass. The electron density widely ranges from 7 to $286 \mathrm{~cm}^{-3}$ across the SFB sample, with a median value of $70 \mathrm{~cm}^{-3}$. The median mass loss rate $\dot{M}_{\text {out }}$ is 14.3 $M_{\odot} \mathrm{yr}^{-1}$ for our SFB sample. At the same time we assess 


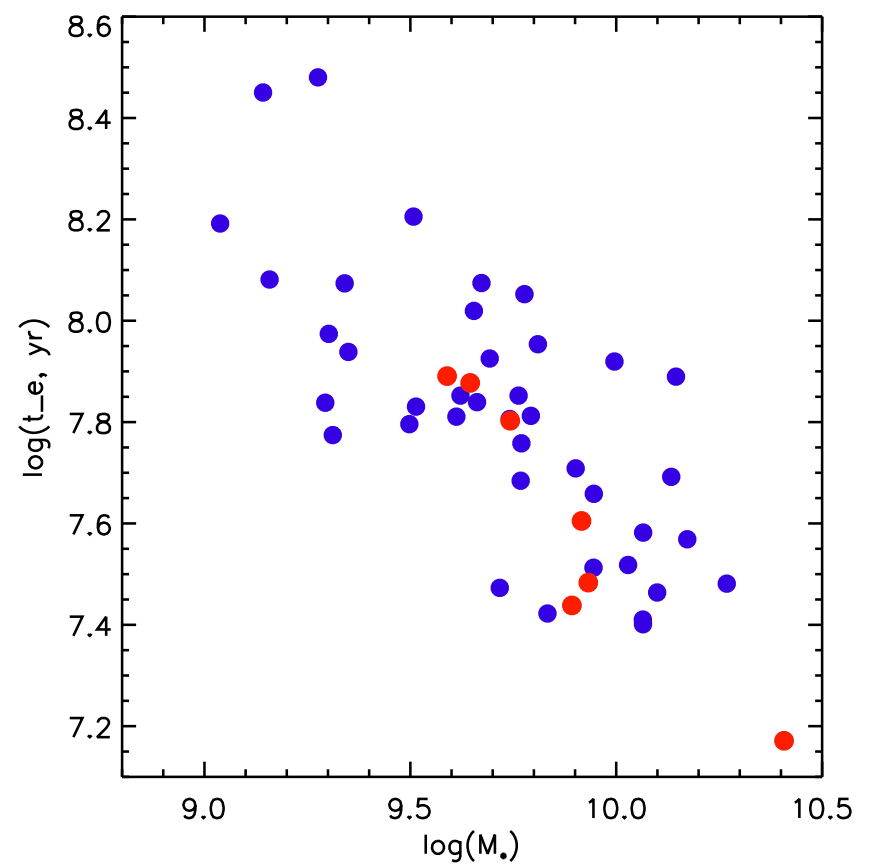

Figure 10. The estimated outflow exhaust time in the main SFB sample (blue bullets) and in seven SFB galaxies with a gas-star counterrotation (red bullets) anticorrelates with the galactic stellar mass.

the ionized gas mass $M_{g c}$ at the center from the volume and density and conclude that the outflow cannot last more than a few hundred Myr: the exhaust time $t_{e}=M_{g c} / \dot{M}_{\text {out }}$ in our SBF sample ranges from 20 to $300 \mathrm{Myr}$ (the median time $t_{e}$ is $65 \mathrm{Myr}$ ). The $t_{e}$ anticorrelates with the galactic stellar mass: it is long in our low mass SFB galaxies and short in the massive galaxies, see Figure 10. The correlation agrees with a natural assumption that the gravitational potential of massive galaxies attracts more circumgalactic gas than for small galaxies. More external gas falls to the central area between the SFB active cycles and supports more intensive star formation during the active cycles in the large galaxies. It is worth noting that the SFB galaxies with counterrotation (red bullets in Figure 10) indicate shorter exhaust time for the outflow, on average, with respect to the other SFB objects. In those cases the external-gas-internalgas interaction can provide more efficient transportation of the gas to the central regions due to a more efficient gas momentum loss, which should shorten the SFB recharging time and the whole duty cycle.

Note that the star formation rate $\dot{M}_{\mathrm{SF}}$ at the center is an order of magnitude less than the outflow rate $\left(\dot{M}_{\text {out }} \gg \dot{M}_{\mathrm{SF}}\right)$, which makes the biconical outflow the principal regulator of the gas mass balance at the central regions. The typical gas infall rate in regular galaxies is comparable to the star formation rate (Chiappini 2009), so a steady infall with a constant rate cannot compensate the gas outflow in the SFB regime. If the gas were supplied in a slow steady infall only, the star formation bicones would show some $1.5 \mathrm{Gyr}$ long "recharging" cycles of the bicone inactivity in large galaxies, and much longer than that in dwarfs. The latter suggests that the SFB activity should be triggered by a bulk accretion of gas (e.g., due to a minor merging).

The triggers for the SFB outflows. The central starburst started in our SFB galaxies less than a few hundred Myr ago, which makes it a temporary phenomenon in galaxies, and requires a triggering event. The gas necessary for feeding the starburst can be of the external or internal origin (Lehnert \& Heckman 1996a). Neither of the SFB galaxies in our sample shows traces of recent or ongoing minor merging or interaction, which excludes recent major mergers.

The accretion of gas from distant companion galaxies or from small, gas-rich satellites to the central area of our SFB galaxies would provide gas supply necessary to start the starburst, and also would explain the gas metallicity distribution in the SFB galaxies. A high fraction of objects with a counterrotation between the gas and stars among the SFB galaxies $(15 \%$, with no counterrotation in the control sample) supports the hypothesis that the gas accretion from satellites can often trigger the SFB ignition. We find that $56 \%$ of our SFB galaxies have large or small companions, or are group members, although we do not see traces of interaction on deep MzLS+BASS images. ${ }^{13}$ The other $17 \%$ of the sample indicate noticeable asymmetry of external isophotes, which suggests that a minor merging took place in the past. This mechanism is also suggested for low mass galaxies with counterrotation between gas and stars, where the central star formation rate is high and young population dominates (Chen et al. 2016).

An alternative mechanism, a rapid internal redistribution of gas in galaxies, e.g., a result of bar-driven gas inflows, may be responsible for driving a large amount of gas to the central regions and for igniting the star formation burst. In this case the internal reasons should be able to increase the rate of transferring the gas to the central region, which may also be caused by enhanced external gas infall (e.g., as a result of an accretion event to the outer regions of galaxy). Both large positive gradients of D4000 and age in the $[\alpha / \mathrm{Fe}]$ indicate the concentration of recently formed young stars to the centers of SFB galaxies. In a combination with large velocity dispersion of gas and stars there it suggests that we may observe the growth of young pseudo-bulges (Lackner \& Gunn 2013), which can be also connected to the secular processes in galactic disks (Kormendy \& Kennicutt 2004; Athanassoula 2005).

While the central outflows remove the gas from the sites of active star formation, we do not see evidence that the gas leaves the galaxies forever, as it has been noticed by Oppenheimer \& Dave (2006), Oppenheimer et al. (2010), Leroy et al. (2015), Emonts et al. (2017), and Rupke (2018). Instead, the gas expelled from the central regions enriches the intergalactic medium and may return back to refuel the starburst-galactic outflow cycle. The properties of many galaxies in our sample resemble those of a well studied nuclear starburst galaxy M82, which helps guess the star formation rates, outflow rates, and the warm gas budget at the centers of our galaxies. Our estimates show that the outflow rates exceed the inflow rates by the order of magnitude. We also expect that our estimates of the gas outflow rates set only the lower limit because the hot gas in the outflow entrains the warm and cold gas components (see e.g., Rupke 2018; Zhang 2018), while our observations are sensitive to the warm component of the gas media only. The intensive mass loss with respect to the gas replenishing rate suggests a temporary nature of the SFB and points at the necessity of prolonged "gas recharging" time. Our criteria for the SFB galaxies selection will help select and study more clear cases of face-on SFB galaxies, in which the kinematics of the outflows can be studied directly from ongoing spectroscopic

\footnotetext{
13 http://legacysurvey.org/pubs/
} 
observations, and the gas mass circulation rate can be estimated in a more straightforward way.

D.B. is partly supported by RSCF grant 19-12-00145. Y.C. acknowledges support from the National Key R\&D Program of China (No. 2017YFA0402700), the National Natural Science Foundation of China (NSFC grants 11573013, 11733002) Y.S. acknowledge support from the National Key R\&D Program of China (No. 2018YFA0404502) and the National Natural Science Foundation of China (NSFC grants 11733002 and 11773013). R.R. thanks CNPq, FAPERGS, and CAPES for financial support.

We thank the anonymous referee for valuable feedback that improved the paper.

SDSS-IV acknowledges support and resources from the Center for High-Performance Computing at the University of Utah. The SDSS website is www.sdss.org.

SDSS-IV is managed by the Astrophysical Research Consortium for the Participating Institutions of the SDSS Collaboration including the Brazilian Participation Group, the Carnegie Institution for Science, Carnegie Mellon University, the Chilean Participation Group, the French Participation Group, Harvard-Smithsonian Center for Astrophysics, Instituto de Astrofísica de Canarias, The Johns Hopkins University, Kavli Institute for the Physics and Mathematics of the Universe (IPMU)/University of Tokyo, Lawrence Berkeley National Laboratory, Leibniz Institut für Astrophysik Potsdam (AIP), MaxPlanck-Institut für Astronomie (MPIA Heidelberg), Max-PlanckInstitut für Astrophysik (MPA Garching), Max-Planck-Institut für Extraterrestrische Physik (MPE), National Astronomical Observatory of China, New Mexico State University, New York University, University of Notre Dame, Observatrio Nacional/ MCTI, The Ohio State University, Pennsylvania State University, Shanghai Astronomical Observatory, United Kingdom Participation Group, Universidad Nacional Autónoma de México, University of Arizona, University of Colorado Boulder, University of Oxford, University of Portsmouth, University of Utah, University of Virginia, University of Washington, University of Wisconsin, Vanderbilt University, and Yale University.

\section{ORCID iDs}

Dmitry Bizyaev (iD https://orcid.org/0000-0002-3601-133X

Yong Shi (i) https://orcid.org/0000-0002-8614-6275

Rogemar A. Riffel (iD https://orcid.org/0000-0003-0483-3723

Namrata Roy (iD https://orcid.org/0000-0002-4430-8846

\section{References}

Athanassoula, E. 2005, MNRAS, 358, 1477

Baldwin, J. A., Phillips, M. M., \& Terlevich, R. 1981, PASP, 93, 5

Bizyaev, D., Walterbos, R. A. M., Yoachim, P., et al. 2017, ApJ, 839, 87

Blanton, M. R., Bershady, M. A., Abolfathi, B., et al. 2017, AJ, 154, 28

Blanton, M. R., Kazin, E., Muna, D., et al. 2011, AJ, 142, 31

Bundy, K., Bershady, M. A., Law, D. R., et al. 2015, ApJ, 798, 7

Burbidge, E. M., Burbidge, G. R., \& Rubin, V. C. 1964, ApJ, 140, 942

Cardelli, J. A., Clayton, G. C., \& Mathis, J. S. 1989, ApJ, 345, 245

Chang, Y.-Y., van der Wel, A., da Cunha, E., \& Rix, H.-W. 2015, ApJS, 219, 8

Chen, Y.-M., Shi, Y., Tremonti, C. A., et al. 2016, NatCo, 7, 1326
Chen, Y.-M., Tremonti, C. A., Heckman, T. M., Kauffmann, G., et al. 2010, AJ, 140, 445

Cheung, E., Bundy, K., Cappellari, M., et al. 2016, Natur, 533, 504

Chiappini, C. 2009, in IAU Symp. 254, The Galaxy Disk in Cosmological Context, ed. J. Andersen, J. Bland-Hawthorn, \& B. Nordstrom (Cambridge: Cambridge Univ. Press), 191

Chisholm, J., Tremonti, C., Leitherer, C., Chen, Y.-M., et al. 2015, ApJ, 811,149

Davies, R., Foster Schreiber, N., Ubler, H., et al. 2019, ApJ, 873, 122

Dib, S., Bell, E., \& Burkert, A. 2006, ApJ, 638, 797

Dopita, M. A., Kewley, L. J., Sutherland, R. S., \& Nicholls, D. C. 2016 Ap\&SS, 361, 61

Drory, N., MacDonald, N., Bershady, M. A., et al. 2015, AJ, 149, 77

Emonts, B. H. C., Colina, L., Piqueras-López, J., et al. 2017, A\&A, 607, 116 Fielding, D., Quataert, E., Martizzi, D., et al. 2017, MNRAS, 470, 39

Gallagher, R., Maiolino, R., Belfiore, F., et al. 2019, MNRAS, 485, 3409

Gorgas, J., Cardiel, N., Pedraz, S., \& Gonzalez, J. J. 1999, A\&AS, 139, 29

Gunn, J, Siegmund, W., Mannery, E., et al. 2006, AJ, 131, 2332

Heckman, T. 2002, in ASP Conf. Proc. 254, Extragalactic Gas at Low Redshift, ed. J. S. Mulchaey \& J. Stocke (San Francisco, CA: ASP), 292

Heckman, T., Alexandrof, R., Borthakur, S., et al. 2015, ApJ, 809, 147

Heckman, T., Armus, L., \& Miley, G. 1990, ApJS, 74, 833

Heckman, T., Lehnert, M., Strickland, D., \& Armus, L. 2000, ApJS, 129, 493

Ho, I. T., Medling, A. M., Bland-Hawthorn, J., et al. 2016, MNRAS, 457, 1257

Kauffmann, G., Heckman, T. M., Tremonti, C., et al. 2003, MNRAS, 346, 1055

Kewley, L. J., \& Dopita, M. A. 2002, ApJS, 142, 35, O2N2

Kewley, L. J., Dopita, M. A., Sutherland, R. S., et al. 2001, ApJ, 556, 121

Kormendy, J., \& Kennicutt, R. C., Jr. 2004, ARA\&A, 42, 603

Krumholz, M. R., \& Burkhart, B. 2016, MNRAS, 458, 1671

Lackner, C. N., \& Gunn, J. E. 2013, MNRAS, 428, 2141

Law, D. R., Cherinka, B., Yan, R., et al. 2016, AJ, 152, 83

Law, D., Yan, R., Bershady, M. A., et al. 2015, AJ, 150, 19

Lehnert, M., \& Heckman, T. 1995, ApJS, 99, 173

Lehnert, M., \& Heckman, T. 1996a, ApJ, 472, 546

Lehnert, M., \& Heckman, T. 1996b, ApJ, 462, 651

Lehnert, M. D., Heckman, T. M., \& Weaver, K. A. 1999, ApJ, 523, 575

Lehnert, M. D., Nesvadba, N. P. H., Le Tiran, L., et al. 2009, ApJ, 699, 1660

Leroy, A. K., Walter, F., Martini, P., et al. 2015, ApJ, 814, 83

Levy, R. C., Bolatto, A. D., Sanchez, S., et al. 2019, arXiv:1905.05196

Lynds, C. R., \& Sandage, A. R. 1963, ApJ, 137, 1005

Martin, C. L., \& Kennicutt, R. C., Jr. 2001, ApJ, 555, 301

McWilliam, A. 1997, ARA\&A, 35, 503

Oppenheimer, B. D., \& Dave, R. 2006, MNRAS, 373, 1265

Oppenheimer, B. D., Dave, R., Keres, D., et al. 2010, MNRAS, 406, 2325

Osterbrock, D. 1989, Astrophysics of Gaseous Nebulae and Active Galactic Nuclei (Mill Valley, CA: Univ. Science Books)

Perez-Montero, E. 2017, PASP, 129, 3001

Pilyugin, L. S., \& Grebel, E. K. 2016, MNRAS, 457, 3678

Riffel, R. A., Nemmen, R. S., Ilha, G. S., et al. 2019, MNRAS, 485, 5590

Roche, N., Humphrey, A., Gomes, J. M., et al. 2015, MNRAS, 453, 2394

Roy, N., Bundy, K., Cheung, E., et al. 2018, ApJ, 869, 117

Rubin, K., Prochaska, J. X., Koo, D. C., et al. 2014, ApJ, 794, 156

Rupke, D. 2018, Galax, 6, 138

Schneider, E. E., Robertson, B. E., \& Thompson, T. A. 2018, ApJ, 862, 56

Shopbell, P. L., \& Bland-Hawthorn, J. 1998, ApJ, 493, 129

Smee, S., Gunn, J. E., Uomoto, A., et al. 2013, AJ, 146, 32

Strickland, D., \& Heckman, T. 2007, ApJ, 658, 258

Tenorio-Tagle, G., \& Muñoz-Tuñón, C. 1998, MNRAS, 293, 299

Thomas, D., Maraston, C., \& Bender, R. 2002, MNRAS, 339, 897

Tremonti, C. A., Heckman, T. M., Kauffmann, G., et al. 2004, ApJ, 613, 898

van der Kruit, P. C., \& Freeman, K. C. 2011, ARA\&A, 49, 301

Veilleux, S., Cecil, G., \& Bland-Hawthorn, J. 2005, ARA\&A, 43, 769

Veilleux, S., Shopbell, P. L., Rupke, D. S., et al. 2003, AJ, 126, 2185

Wake, D. A., Bundy, K., Diamond-Stanic, A. M., et al. 2017, AJ, 154, 86

Worthey, G., \& Ottaviani, D. L. 1997, ApJS, 111, 377

Yan, R., Bundy, K., Law, D. R., et al. 2016, AJ, 152, 197

York, D., Adelman, J., Anderson, J., et al. 2000, AJ, 120, 1579

Yu, X., Shi, Y., Chen, Y., et al. 2019, MNRAS, 486, 4463

Zhang, D. 2018, Galax, 6, 114 